\title{
DYNAMICAL REFERENCE FRAMES IN THE PLANETARY AND EARTH-MOON SYSTEMS
}

\author{
E. M. Standish and J. G. Williams \\ Jet Propulsion Laboratory \\ California Institute of Technology \\ 4800 Oak Grove Drive \\ Pasadena, CA 91109 USA
}

\begin{abstract}
We summarize our previous estimates of the accuracies of the ephemerides. Such accuracies determine how well one can establish the dynamical reference frame of the ephemerides. Ranging observations are the dominant data for the inner four planets and the Moon: radar-ranging for Mercury and Venus; Mariner 9 and Viking spacecraft-ranging for the Earth and Mars; lunar laser-ranging for the Moon. Optical data are significant for only the five outermost planets. Inertial mean motions for the Earth and Mars are determined to the level of $0.003 / c t y$ during the time of the Viking mission; for Mars, this will deteriorate to $0.01 / c t y$ or more after a decade or so; similarly, the inclination of the martian orbit upon the ecliptic was determined by Viking to the level of 0:001. Corresponding uncertainties for Mercury and Venus are nearly two orders of magnitude larger. For the lunar mean motion with respect to inertial space, the present uncertainty is about 0"04/cty; at times away from the present, the uncertainty of $1 " / \mathrm{cty}^{2}$ in the acceleration of longitude dominates. The mutual orientations of the equator, ecliptic and lunar orbit are known to 0."002. The inner four planets and the Moon can now be aligned with respect to the dynamical equinox at a level of about 0.005 .
\end{abstract}

\section{Introduction}

By estimating the accuracies of the planetary and lunar ephemerides themselves, the authors have estimated the accuracy of the inherent dynamical reference frame (Williams and Standish, 1989, hereafter referred to as Paper I). The present paper gives a summarized version of the former estimations, briefly listing the observational data in order to emphasize the variety of data types, presenting a table of the estimated accuracies and noting some recent ephemeris improvements. An attempt is again made to illustrate why the mean motions of the inner planets and the Moon are determined almost exclusively by ranging data, not by optical observations. Finally, the paper discusses a long-known discrepancy: mean motions determined by fitting to only optical observations differ by 1 "/century from those determined by fitting to ranging data.

The observational data are listed in Section 2; Section 3 notes recent improvements to the ephemerides of the outer planets; the determination of inertial mean motions using ranging data is discussed in Section 4; Section 5 presents a summary of the error estimates derived from Paper I and Section 6 discusses the 1 "/cty discrepancy. 


\section{The Observational Data}

The most important ingredient in the process of creating modern-day ephemerides is the collection of the observational data: accuracy, variety and coverage. With modern computers, there is no longer any problem correctly integrating the equations of motion: no worry about non-converging expansions, neglected terms, truncated series, etc. Numerical integration programs have been tested; they provide sufficient accuracy. Also, it seems safe to assume that our equations of motion properly describe the physical laws of gravitation and that all of the significant forces which affect the motions of the planets and Moon are known.

Table 1 lists the observational data now being fit to generate ephemerides. During the present decade, in preparation for the Voyager encounters, newer and more accurate observations of the five outermost planets have produced significant improvements to those ephemerides. These are discussed in the next Section.

The acquisition and utilization of observational data continues to be the most vital part of the ephemeris creation process.

Table 1. The sources of the observational data.

\begin{tabular}{|c|c|c|c|c|c|c|c|c|c|c|}
\hline & Sun & Mer & Ven & Mar & Jup & Sat & Ura & Nep & $\mathrm{Plu}$ & Moon \\
\hline Optical transits & 1911- & $\mathbf{M}$ & $\mathbf{V}$ & $\mathbf{M}$ & $\mathbf{J}$ & $S$ & $\mathrm{U}$ & $\mathbf{N}$ & & \\
\hline Photoelectric transits & $1982-$ & & & $\mathbf{M}$ & $\mathbf{J}$ & $S$ & $\mathrm{U}$ & $\mathbf{N}$ & $\mathbf{P}$ & \\
\hline Astrolabe & 1969- & & & $\mathbf{M}$ & $\mathbf{J}$ & $S$ & $\mathrm{U}$ & & & \\
\hline Radar ranging & 1964- & $\mathbf{M}$ & $\mathrm{V}$ & $\mathbf{M}$ & & & & & & \\
\hline Mariner 9 Ranges & $1971-72$ & & & $\mathbf{M}$ & & & & & & \\
\hline Mariner 10 Ranges & $1974-75$ & $\mathbf{M}$ & & & & & & & & \\
\hline Viking Lander Ranges & $1976-82$ & & & $\mathbf{M}$ & & & & & & \\
\hline Radio Astrometry & 1983- & & & & $\mathbf{J}$ & $S$ & $\mathrm{U}$ & $\mathbf{N}$ & & \\
\hline Ring Occultations & $1977-$ & & & & & & $\mathrm{U}$ & & & \\
\hline Disk Occultations & $1968-$ & & & & & & & $\mathbf{N}$ & & \\
\hline Pioneer Tracking Data & $1973-80$ & & & & $\mathbf{J}$ & $S$ & & & & \\
\hline Voyager Tracking Data & $1979-89$ & & & & $\mathbf{J}$ & $S$ & $\mathrm{U}$ & $\mathbf{N}$ & & \\
\hline Pluto Astrometry & $1914-$ & & & & & & & & $\mathbf{P}$ & \\
\hline Lunar Laser Ranging & 1969- & & & & & & & & & $\mathbf{M}$ \\
\hline
\end{tabular}

\section{Outer Planet Ephemerides}

It is apparent in Table 1 that there are now newer and more accurate types of observational data than what were available just a decade ago. This is especially true for the outer planets where a concentrated effort was made for the Voyager encounters with the Jovian planets. Indeed, there are significant differences between the ephemerides DE202, JPL's latest, and its predecessor at JPL, DE200, the standard planetary ephemeris of the Astronomical Almanac. Since DE202 is believed to be much more accurate than DE200 for the present epoch, most ( $80+$ percent?) of the difference 
may be attributed to errors in DE200.

The differences, DE202-DE200, are plotted in a recent paper by Standish (1989). For Jupiter, the right ascension varies over the planet's 12 -yr period between -0 ". 1 and -0.2 throughout the century. For Saturn, the right ascension also varies over the 30-yr period, but in addition, drifts down to -0"25 at present. The difference for Uranus is small through the first half of the century, but reaches nearly -0.5 by the year 2000 . For Neptune, the error is +0.6 for 1900 and is near -1.0 by 2000 . The error for Pluto exceeds +2.0 by the end of the century and is rapidly increasing - not surprising since this is the first time that JPL has fit the orbit of Pluto to observations. The declination errors are generally periodic and smaller than those of right ascension.

For the four Jovian planets, consistency of all of the new observations taken during the present decade indicate that over that time-span, DE202 has position errors of 0.05 or less. For Pluto, observed only photographically to date, the ephemeris is expected to have an uncertainty of about 0.5 during the present era, due mainly to catalogue errors. This uncertainty may decrease in future ephemerides, now that photoelectric transit observations of Pluto are being obtained at La Palma (Morrison et al., 1990).

\section{Inertial Mean Motions}

There are a number of explanations designed to show why inertial mean motions are determined almost exclusively from the ranging data; why the mean motions of the four inner planets and the Moon are almost entirely independent of the optical observations (Standish 1982, 1985, 1988; Paper I). We give one more here.

Consider the Newtonian two-body problem: True, the distances are invariant under a rotation of the ellipse. However, a rotating ellipse is not a possible Newtonian two-body motion.

The actual case of the solar system is similar; the planetary orbits are nearly non-rotating ellipses. The departures from Keplerian motion, for the most part, are small and accurately computed. It is the accuracy of computing these departures which determines the major uncertainties in the mean motions for the moon and planets; these were discussed in Paper I.

In the Newtonian case, the lunar range is given by

$$
r=a-a e \cos E \approx a-a e \cos n(t-T)
$$

i.e., there is a constant term, an amplitude, a phase and a period (inertial mean motion). Given a reasonable span of observations, each of these parameters can be determined to an accuracy comparable to or better than the 3 centimeter accuracy of the present data. For the actual case, the non-Keplerian features of the lunar motion include solar and planetary perturbations, general relativity, the tidal deceleration in longitude and the precession of the perigee and node. As shown in Paper I, the greatest uncertainty in the lunar motion is in the perigee's precession, 0."04/cty, for times near the center of the data span; for remote times, the tidal deceleration uncertainty of $1 " / \mathrm{cty}^{2}$ dominates.

The case for planetary ranging is analogous, though here the ranging occurs between bodies moving on two ellipses instead of one. Nevertheless, the same principle applies, as described in Paper I. The observational data for Earth and Mars are dominated by the six years of ranging to the Viking Landers at 10 meter accuracy. The major uncertainty in the motion with respect to an inertial frame is due to uncertainties in Mars' perihelion rate due to mass uncertainties of the planets. When the motion is extrapolated decades to centuries beyond the span of the ranging data, the dominant error 
source becomes the long-period perturbations from many asteroids whose masses are not known better than a factor of about two.

\section{Ephemeris Error Estimates}

In Paper I, an analysis of the ephemeris parameter uncertainties was made by considering the accuracies of the relevant observational data and by considering how sensitive such observations are to changes in each of the parameters. Those results are used here in producing Table 2, where the estimates are intended to be realistic uncertainties. We also include our estimates of the orbits of the outer planets, realizing that the uncertainty of extrapolating into the future is largest for the outermost planets.

Table 2. Estimated ephemeris errors.

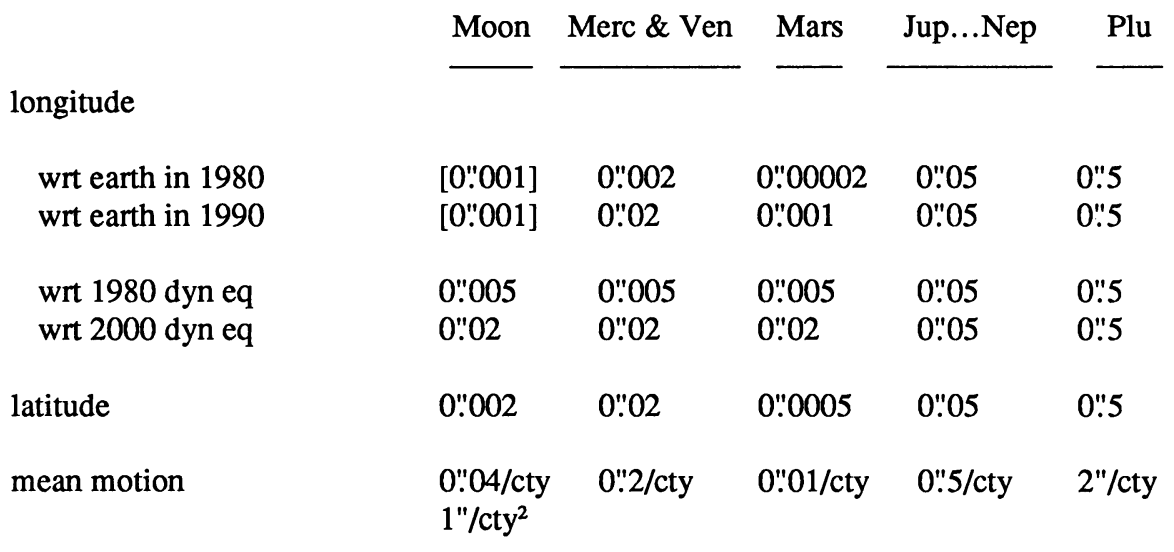

\section{Optical Problem}

Something is wrong with the optical transit observations and/or with the reference systems with which they are reduced.

The optical residuals for the Sun and inner planets show secular drifts in right ascension of approximately one arcsecond per century. An example is given in Figure 1a showing the 20th century solar residuals of observations taken with the USNO 6" and 9" transit circles. Without the presence of ranging data, the drift would be removed by the least squares adjustment of the orbits, primarily by changes in the semi-major axes. This is essentially what happens with the outer five planets, for which there are no ranging measurements. However, in the presence of the dominating range data, the orbits for the inner four planets are adjusted primarily to the ranges, not to the optical observations. 
This problem has been recognized for many years (Stumpff and Lieske, 1984; Seidelmann et al., 1985; Seidelmann, 1986). The source, however, remains unknown. Two things are certain: the error does not come from the newly adopted value of precession (Fricke, 1971); the error does not come from the mean motions of the inner planets in the modern ephemerides. The first assertion is valid since both lunar laser ranging and VLBI have now verified that the error in Fricke's value of precession is no more than about $0.3 /$ cty. For the second assertion, we here perform an experiment.
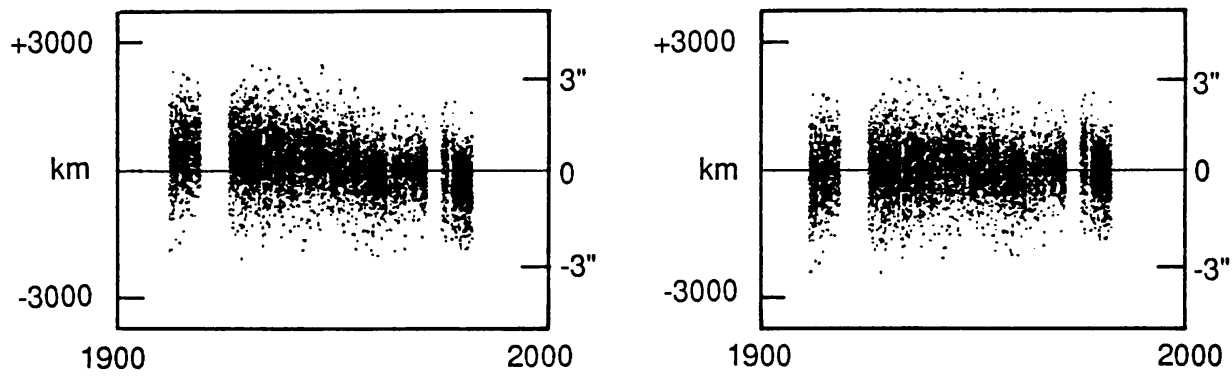

Figures 1a and 1b. The right ascension residuals of the Sun from the USNO 6-inch and 9inch meridian circle observations. A downward slope of about 1"/cty is noticeable in Figure $1 \mathrm{a}$, where the residuals are plotted with respect to a present day ephemeris. The slope is gone in Figure 1b where the Earth's mean motion has been forced to change by 1"/century.
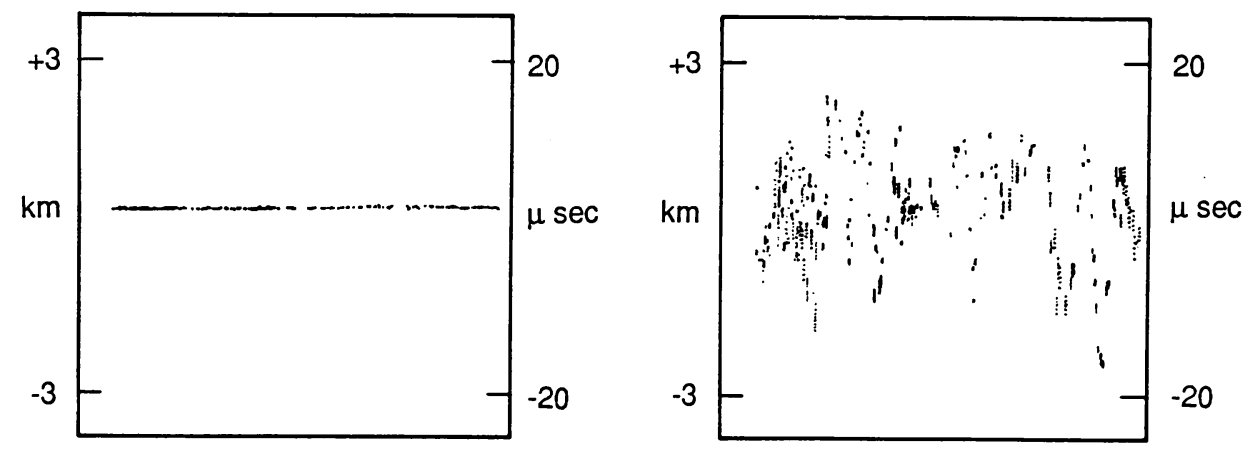

Figures 2a and $\mathbf{2 b}$. The range residuals from the Viking Lander on Mars. Figure 2a shows the residuals from a present-day ephemeris. Figure $2 b$ shows the residuals from an ephemeris which has been forced to fit a change of 1"/century in the Earth's mean motion. 


\subsection{Forcing the Earth's Mean Motion to Fit the Optical Data}

In order to illustrate how sensitive the mean motions are to ranging data, we artificially force a change of 1 "/cty into the mean motion of the Earth and then perform a full least squares adjustment of all of the other ephemeris parameters.

Figures 1a and $1 \mathrm{~b}$ show the right ascension residuals of the Sun before and after forcing the Earth's mean motion. One can detect the left-to-right downward slope of about 1"/cty in Figure 1a as it appears in present-day ephemerides; in Figure 1b, the slope is gone since the Earth's mean motion has been adjusted artificially. The scale shows that the differences in question correspond to the level of a few hundred kilometers; the differences are not blatantly obvious in these residuals.

In contrast, Figures $2 \mathrm{a}$ and $2 \mathrm{~b}$ show the corresponding residuals of the Viking Lander ranging where the change is gigantic. Previous residuals of 10 meters can no longer be fit better than a full kilometer; further, they would obviously become even worse if extrapolated beyond the six-year interval. This fit is completely unacceptable.

Just as striking are the values of the ephemeris parameters in the attempted adjustment. Some of these are given in Table 3. These results are nonsense: the mass of Ceres quintupled, the masses of

Table 3. The disastrous consequences of forcing the Earth's mean motion to change by 1 /century. Shown are the resultant values for the solar system scale parameter, the Sun/ planet-system mass ratios, the masses of the three major asteroids [in $\mathrm{au}^{3} / \mathrm{day}^{2} \times 10^{-13}$ ], the densities of the S-type and C-type asteroids and the locations of the Deep Space Network radio antennas.

\begin{tabular}{|c|c|c|c|c|}
\hline Parameter & Present Value & Std. Dev. & Forced Change & Ratio \\
\hline scale $(\mathrm{m} / \mathrm{au})$ & 149597870660 & 20 & -1340 & 67 \\
\hline Sun/Venus & 408523.5 & 0.5 & +8 & 16 \\
\hline Sun/Jupiter & 1047.3491 & 0.0002 & +0.0150 & 75 \\
\hline Sun/Saturn & 3497.90 & 0.02 & +4.50 & 225 \\
\hline Sun/Uranus & 22902.96 & 0.02 & -18 & 900 \\
\hline Sun/Neptune & 19412.25 & 0.06 & -7000 & 350000 \\
\hline GM(Ceres) & 1.75 & 0.09 & +7.8 & 87 \\
\hline GM(Pallas) & .32 & 0.05 & -1.0 & 20 \\
\hline GM(Vesta) & .41 & 0.08 & -1.9 & 24 \\
\hline S-class density & 2.0 & 0.5 & -28 & 56 \\
\hline C-class density & 2.0 & 0.5 & -31 & 62 \\
\hline DSN longitudes $[\mathrm{cm}]$ & - & 30 & 100000 & 3300 \\
\hline spin radii $[\mathrm{cm}]$ & - & 30 & 100000 & 3300 \\
\hline $\mathrm{z}$-heights $[\mathrm{cm}]$ & - & 50 & 200000 & 4000 \\
\hline
\end{tabular}


Pallas and Vesta became negative, etc. Most of the changes to the parameters are on the order of 100 times their presently accepted standard deviations.

The result is clear: there is no way that the mean motion of the Earth in modern ephemerides can be in error by 1 "/cty.

An error of $1 " /$ cty is certainly greater than one hopes for the optical system; however, it is completely intolerable for modern planetary ranging.

\section{Conclusions}

The ephemerides continue to improve as more and newer data measurements are acquired. In particular, over the past decade, substantial improvements have been made to the outer planet ephemerides. Corresponding refinements to the ephemerides of the inner planets and the Moon are expected within the next year.

A number of different methods have been considered for estimating the errors in the modern-day ephemerides. They seem to be consistent with each other; their results were presented here.

Not all of the observational data sets are consistent, however. In particular, there is still the puzzling drift in the optical residuals of about 1 "/century for the inner bodies of the solar system.

The forcing of a 1"/century change in the mean motion of the Earth required the other ephemeris parameters to change by about 100 times their standard deviations in a futile attempt to re-fit the data. This tends to indicate that the mean motion of the Earth has been established to a level of about 0."01/ century.

One must conclude that there is something wrong with the optical data and/or the reduction of it: not precession certainly, but possibly the equinox drift; perhaps not the values themselves, but possibly where and when the parameters are applied in the reduction processes. It is too early to speculate further; it is intended that this will be investigated soon.

Acknowledgment. The work described in this paper was carried out by the Jet Propulsion Laboratory, California Institute of Technology, under contract with the National Aeronautics and Space Administration.

\section{References}

Fricke, W.: 1971, “A Rediscussion of Newcomb's Determination of Precession”, Astron. Astrophys. 13, 298-308.

Fricke, W.: 1982, "Determination of the Equinox and Equator of the FK5", Astron. Astrophys. 107, L13-L16.

Morrison, L.V., Helmer, L., Fabricius, C., Einicke, O., Quijano, L., Muinos, J.L., Argyle, R.W.: 1990, "Optical Reference Frame Defined by Carlsberg Meridian Catalogues for the Years 1984-1987", these proceedings.

Seidelmann, P.K.: 1986, "Unsolved Problems of Celestial Mechanics — The Solar System", Cel., Mech., 39, 141-146.

Seidelmann, P.K., Santoro, E.J. and Pulkkinen, K.F.: 1985, Systematic Differences between Planetary Observations and Ephemerides", in Dynamical Astronomy, V. Szebehely and B. Balazs, Eds., University of Texas Press, Austin, 55-65. 
Standish, E.M.: 1982, “The JPL Planetary Ephemerides”, Cel., Mech. 26, 181-186.

Standish, E.M.: 1985, "On the Orientation of Ephemeris Reference Frames”, Cel., Mech. 37, 239-242.

Standish, E.M.: 1988, “Celestial Reference Frames: Definitions and Accuracies”, in The Impact of VLBI on Astrophysics and Geophysics (M.J. Reid and J.M. Moran, Eds.), D. Reidel, publ., 309-315.

Standish, E.M.: 1989, "An approximation to the outer planet ephemeris errors in JPL's DE200", Astron. Astrophys, in press.

Stumpff, P. and Lieske, J.H.: 1984, "The Motion of the Earth-Moon System in Modern Tabular Ephemerides", Astron. Astrophys., 130, 211-226.

Williams, J.G. and Standish, E.M.: 1989, "Dynamical Reference Frames in the Planetary and EarthMoon Systems", in Reference Frames (J. Kovalevsky, I.I. Mueller and B. Kolaczek, eds.) Kluwer Academic Publishers, Dordrecht, 67-90.

\section{Discussion}

KRASINSKY: We have obtained similar results on the inconsistency of planetary mean motions determined from radar and optical observations. In our opinion the discrepancy may be explained by secular errors in the ephemeris time scale before 1959. There are indications that these errors do exist (from analyses of Mercury and Venus solar transits). These errors influence the mean motions in the same way as an error in the precessional constant.

STANDISH: In order to produce an error of 1"/century in the solar residuals, the ET-UT tables would have to be in error by 24 time seconds/century. That is too large for most people to accept.

HuGHEs: As is well known, observing the Sun is difficult. I would comment that the new observing system on our instrument in New Zealand has increased our ability to observe day-stars. We commonly observe up to 50 stars in a day including azimuth stars. This certainly will help with the perennial problem of relating day and night observations.

STANDISH: Those observations certainly will be instructive in a number of ways. However, I do not believe that the $1 " /$ century is a jroblem of data observation; rather, one of data reduction.

KAPLAN: Could you comment on the possibility-or impossibility-of significant amounts of unobserved mass in the solar system? In particular, a tenth planet?

Standish: Yes, I often make such comments. Certainly, there is nothing to say that there could not be significant amounts of mass outside of Pluto, even a concentration large enough to be a planet. However, certain regions seem to be excluded by the different searches (Tombaugh, Kowal, IRAS, etc.).

Further, there is nothing in the observational planetary data which needs Planet $\mathrm{X}$ to explain it. With a 20th century error as gigantic as 1 "/century, it seems silly to invoke a Planet $\mathrm{X}$ to explain only a couple of tenths of an arcsecond from the 19th century data. What's even worse, the newer data are greatly improved in comparison to the older data which are fraught with known systematic errors. 
SEIDELmanN: I predict that in ten years the observations of Neptune and Pluto will deviate from the ephemerides as they have each time in the past.

STANDISH: I agree; accurate extrapolation can not be expected. We have less than a period's worth of observations for these planets, and the observations that we do have contain systematic errors.

There is no question, though; we continue to need the optical observations, especially those of the outer planets.

KLIONER: What is the present accuracy of the barycentric position of the Sun in kilometers?

STANDISH: The former $(0.5 \%)$ error in the mass of Neptune used to be the dominant error in the position of the barycenter, amounting to about $1200 \mathrm{~km}$. Now, after the Voyager determination, the uncertainties due to the uncertainties of the masses of Jupiter through Pluto are $0.1,2.4,0.1,0.7$ and $10.4 \mathrm{~km}$, respectively. 